\title{
BMJ Open Patient-reported outcomes and associations with pleural effusion in outpatients with heart failure: an observational cohort study
}

\author{
Guri H Gundersen, ${ }^{1}$ Tone M Norekvål, ${ }^{2,3}$ Torbjørn Graven, ${ }^{1}$ Hilde H Haug, ${ }^{1}$ \\ Kyrre Skjetne, ${ }^{1}$ Jens $O$ Kleinau, ${ }^{1}$ Lise T Gustad, ${ }^{1,4,5}$ Håvard Dalen ${ }^{1,6,7}$
}

To cite: Gundersen $\mathrm{GH}$ Norekvål TM, Graven T, et al. Patient-reported outcomes and associations with pleural effusion in outpatients with heart failure: an observational cohort study. BMJ Open 2017;7:e013734. doi:10.1136/bmjopen-2016013734

- Prepublication history for this paper is available online. To view these files please visit the journal online (http://dx.doi.org/10.1136/ bmjopen-2016-013734).

Received 4 August 2016 Revised 9 December 2016 Accepted 14 February 2017

CrossMark

For numbered affiliations see end of article.

Correspondence to Dr Guri H. Gundersen; guri@ntebb.no

\section{ABSTRACT}

Objectives: We aimed to study whether patientreported outcomes, measured by quality of life $(\mathrm{Q} o \mathrm{~L})$ and functional class, are sensitive to pleural effusion (PLE) in patients with heart failure (HF), and to study changes in QoL and functional class during follow-up of PLE.

Methods: A cohort of 62 patients from an outpatient HF clinic was included. The amount of PLE was quantified using a pocket-sized ultrasound imaging device. Self-reports of QoL and functional class were collected using the Minnesota Living with Heart Failure Questionnaire (MLHFQ) and the New York Heart Association (NYHA) functional classification.

Results: At baseline, 26 (42\%) patients had PLE of which 19 (31\%) patients had moderate to severe amounts of PLE. Patients with no to mild PLE had a lower MLHFQ score (mean 42, SD 21) compared with patients with a moderate to severe amount of PLE (mean 55, SD 24), $p=0.03$. For 28 patients (45\%) with follow-up data, we observed a linear improvement of the MLHFQ-score (3.2, 95\% Cl 1.2 to 5.1) with each centimetre reduction of PLE. Correspondingly, patientreported NYHA-class followed the same pattern as the MLHFQ-score.

Conclusions: Our study indicates that patient-reported outcome measures as MLHFQ may be sensitive tools to identify patients with $\mathrm{HF}$ at highest risk of symptomatic PLE and that treatment targeting reduction of PLE during follow-up is essential to improvement of QoL and functional capacity of outpatients with HF.

Trial registration number: NCT01794715; Results

\section{INTRODUCTION}

Pleural effusion (PLE) is a common sign associated with congestion and worsening of heart failure $(\mathrm{HF})^{1}$ and is often followed by dyspnoea. ${ }^{2}$ However, the degree of dyspnoea may not correlate with the amount of PLE ${ }^{34}$ and screening for PLE is often restricted to patients with HF with clinical signs of PLE.

\section{Strengths and limitations of this study}

- Patient-reported outcome measures of quality of life (QoL) and objective measurements of pleural effusion (PLE) by point-of-care ultrasound was followed up repeatedly in a heart failure (HF) outpatient clinic.

- QoL is increasingly emphasised in treatment strategies and point-of-care ultrasound is recently recommended as a tool to identify PLE in European Society of Cardiology guidelines.

- The study was conducted at one single HF outpatient clinic and the sample size was small. However, the presented population is previously shown to be comparable with studies from other HF clinics with regard to HF severity, age, sex, New York Heart Association class and comorbidity.

It is well known that patients with HF experience significant reductions in quality of life (QoL). ${ }^{5}$ Thus, initiatives to find treatment strategies that improve QoL have become an important objective in the follow-up of patients with $\mathrm{HF}^{7}$ In cancer care, initiatives to reduce PLE seem to be an essential palliative step, which has proven to improve QoL and decrease symptom burden without particular side effects. ${ }^{8}{ }^{9}$ However, poor self-reported QoL or functional capacity are not conventionally used indicators for screening and treatment of PLE in patients with HF. This may be due to the fact that it is largely unknown if QoL or functional class is associated with the presence of PLE in patients with HF. Further, to the best of our knowledge, no previous studies have investigated whether QoL and functional class improves in patients with $\mathrm{HF}$ when PLE is successfully treated. Thus, we aimed to study whether patient-reported outcomes (QoL and functional class) are sensitive to PLE, and to study changes in QoL and functional class during follow-up of PLE. 


\section{METHODS}

\section{Study population and design}

This was a prospective follow-up study with inclusion of 62 patients admitted to the outpatient HF clinic at the non-university Levanger Hospital, Norway between 15 April and 21 June 2013. ${ }^{10} 11$ The sample size was determined in order to validate the feasibility and reliability of point-of-care ultrasound examination. ${ }^{10}{ }^{11}$ The HF diagnosis was confirmed according to the European Society of Cardiology (ESC) guidelines by clinical examination, ${ }^{12}$ medical history and echocardiographic examination by one of four experienced cardiologists. ${ }^{10} 11$

All patients provided their written informed consent to participate. The exclusion criteria were inability or unwillingness to consent or HF worsening requiring hospital admission at entry. The study observed patientreported QoL in the follow-up of a clinical trial regarding feasibility and reliability of point-of-care ultrasound in order to determine PLE (ClinicalTrials.gov: ID: NCT01794715). Data regarding feasibility and influence of point-of-care ultrasound have been recently published. ${ }^{10}{ }^{11}$ The observational part of the study was conducted according to the Second Declaration of Helsinki.

\section{Follow-up}

The follow-up of patients at the outpatient HF clinic was managed by two nurses specialised in cardiovascular and intensive care, working in close cooperation with four cardiologists. Briefly, the visits included patient education, self-management counselling and optimisation of the treatment for HF according to the 2012 ESC HF guidelines $^{12}$ in order to reduce the amount of PLE and improve the functional capacity, QoL and prognosis of the patients. None of the patients with HF needed therapeutic drainage of PLE. The follow-up schedule of the outpatient HF clinic was individualised depending on the condition of the patients. The number of visits ranged from one to four visits during the study period. The final follow-up was defined as the visit when the condition of the patient was satisfactory and stable. Twenty-eight $(45 \%)$ patients had a final follow-up visit in the study period (figure 1).

\section{Measurements}

\section{Measurement of PLE}

The nurses received a short, but dedicated training programme in focused point-of-care ultrasound examinations of the pleural cavities. The high feasibility and excellent reliability for quantification of PLE by point-of-care ultrasound performed by the nurses are comprehensively described in a recent publication. ${ }^{10}$ Ultrasound examinations were performed using the Vscan (GE Ultrasound, Horten, Norway) at each visit. With the transducer placed in the intercostal space, the liver and spleen were used as reference points to identify the diaphragm of the right and left hemithoraces, respectively. During quiet breathing, the posterior chest was scanned along the paravertebral, scapular, posterior

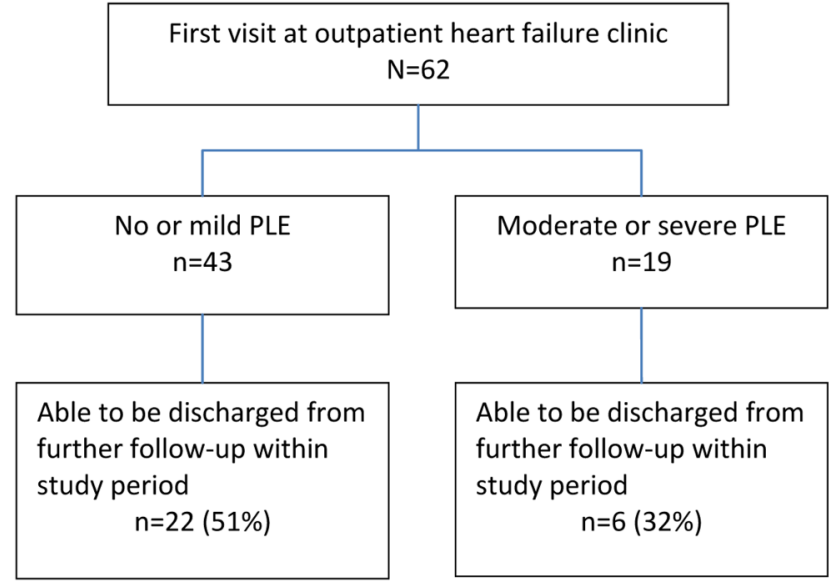

Figure 1 Shows the flow chart of study recruitment and follow-up. The follow-up schedule of the outpatient HF clinic was individualised depending on the condition of the patients. The number of visits ranged from one to four visits during the study period. The final follow-up was defined as the visit when the condition of the patient was satisfactory and stable.

Twenty-eight (45\%) patients had a final follow-up visit in the study period. HF, heart failure.

and medial axillary lines, continuously focusing on the diaphragm as a reference point. PLE was assessed in the costodiaphragmatic angle by assessing the dimension between the diaphragm and the lung surface (measured in the middle between the transducer and the mediastinum). The amount of PLE was categorised in four groups as (1) no PLE, (2) insignificant when present in the costodiaphragmatic angle only, (3) small to moderate when the measurement as described above was $<3 \mathrm{~cm}$ and (4) significant when the measurement was $\geq$ $3 \mathrm{~cm}$. We also dichotomised the PLE measures as no to mild (groups (1) and (2)) and moderate to severe (groups (3) and (4)).

\section{Patient-reported outcome measures}

The patients completed the questionnaires before the clinical examination at the baseline visit and at the final follow-up visit as recommended for patient-reported outcomes. ${ }^{13}$

The functional class was self-reported according to the New York Heart Association (NYHA) classification ${ }^{14}$ on the validated Norwegian version of this instrument. ${ }^{15}$

QoL was reported by the validated Minnesota Living with Heart Failure Questionnaire (MLHFQ), which quantifies how patients with HF perceive their own QoL associated with the HF symptoms and treatment the preceding month. The questionnaire consists of 21 items where the patients are asked to rate how each item prevents them from living the life they desire using a sixpoint Likert scale from 0 to $5(0=$ not at all, $5=$ all the time). The MLHFQ items include: physical HF symptoms (dyspnoea, fatigue, peripheral oedema and sleeping difficulties); psychological HF symptoms (anxiety and depression) and social/functional impairment due 
to HF (walking, climbing stairs, work, household or labour, need to rest, going places away from home, doing things with family and friends, eating, concentration, memory, loss of self-control, being a burden to others and sexual activity). The total score range from 0 to105, where the higher the score, the poorer the QoL. A reduction of 5 units or more in the MLHFQ-score by treatment is considered as clinically meaningful. ${ }^{16}$ We defined a MLHFQ-score $>40$ to be an indicator for poor QoL as this cut-off includes the patients that scores in the upper quartile of the moderate QoL category. ${ }^{17}$

\section{Clinical examination, blood tests and medications}

The specialised nurses examined the patients at all visits. The clinical examinations included ECG (sinus rhythm or not), measurement of the blood pressure $(\mathrm{mm} \mathrm{Hg})$, heart rate (beats $/ \mathrm{min}$ ) and weight (to nearest $0.5 \mathrm{~kg}$ ). ${ }^{10}{ }^{11}$

Blood samples were collected at each visit and analysed at the hospital's International Electrotechnical Commision (IEC) 17025-accredited laboratory. Creatinine was analysed by an enzymatic method developed by Abbott and N-terminal pro-brain natriuretic peptide (NT-proBNP) by electrochemiluminescence immunoassay (ECLIA). Estimated glomerulus filtration rate (e-GFR) was calculated by the chronic kidney disease epidemiology collaboration (CKD-EPI) formula. ${ }^{18}$

The use of $\beta$-blockers was coded as 0 (no use) or 1 (use of) and the same coding was applied for the prescription of ACE inhibitors and angiotensin receptor blockers (ARBs). For diuretics, we recalculated the doses of furosemide, hydrochlorthiazide and bendrofumethiazide to equivalent doses of bumetanide. In the analyses, $40 \mathrm{mg}$ furosemide, $12.5 \mathrm{mg}$ hydrochlorthiazide and $2.5 \mathrm{mg}$ bendrofumethiazide were each recalculated to $1 \mathrm{mg}$ bumetanide. ${ }^{11}$

\section{Statistical analysis}

Comparisons of the baseline characteristics between the participants with no to mild PLE and the participants with moderate to severe PLE and between those participants that self-reported a MLHFQ-score $\leq 40$ compared with those who scored higher were performed by t-test for continuous variables and $\chi^{2}$-test for categorical data. One-way analyses of variance were used in order to assess the differences between MLHFQ-scores in the different categories of PLE and NYHA-classes. For analyses of trend, we treated the PLE categories and NYHA-class as continuous variables in a logistic regression model. Pearson correlation was used to investigate the correlation between patient-reported NYHA-class and MLHFQ-score. Sensitivity, specificity, positive predictive and negative predictive values of the MLHFQ cut-off to detect PLE were calculated at baseline.

Generalised estimating equation with robust SEs and exchangeable correlation structure and inbuilt correction for age was used to model the change of the MLHFQ-score associated with change of PLE over time. For NYHA-class, we used the log link function of generalised estimating equation. In our second model, we adjusted for sex. In the third to seventh model, we added potential confounders ( $\beta$-blockers, bumetanide-equivalent diuretic doses and ACE inhibitors/ARBs, change in weight and change of systolic blood pressure). These variables were added one by one to model 1 , as the sample size was limited. We used a two-way graph based on linear regression to model the change in MLHFQ-score by change in centimetre $(\mathrm{cm})$ PLE. We investigated the potential effect modification by sex and defined the critical $\mathrm{p}$ value to be $<0.10$ for the interaction term. For all other analyses we considered $\mathrm{p}<0.05$ as significant. Statistical analyses were performed in Stata SE/13.1 for windows (@ Stata Corp LP, Collage Station, Texas, USA).

\section{RESULTS}

Table 1 shows the baseline characteristics of the patients $(n=62)$ by PLE amount at baseline. As expected, the group with no or mild PLE had lower NT-proBNP at baseline than the group with moderate to severe PLE. There was a higher proportion of patients with sinus rhythm (49\%) in the group with no to mild PLE compared with the group with moderate to severe PLE (21\%). However, the two groups were comparable in age, sex, blood pressure, heart rate and the use of HF medication (all $\mathrm{p}>0.1$ ). Self-reported MLHFQ-score $>40$ at baseline was associated with higher NYHA-class compared with MLHFQ-score $\leq 40(\mathrm{p}<0.001)$ (data not shown).

As shown in figure 2, the patient-reported MLHFQ-score at baseline depended on the PLE quantity $(p=0.02)$. Approximately half of the patients with no PLE and most patients with moderate-to severe PLE had a MLHFQ-score $>40$. The mean MLHFQ-score in the patients with no or mild PLE was 42 (SD 21) compared with 55 (SD 24) in the patients with moderate to severe PLE $(\mathrm{p}=0.03)$.

As shown in figure 3, self-reported NYHA-class and MLHFQ-score correlated well at baseline $(\mathrm{r}=0.63$, $\mathrm{p}<0.001)$. MLHFQ-score was higher with more severe symptoms (NYHA-class 1 to 4 ) with $\mathrm{p}=0.001$ for trend.

A MLHFQ-score $>40$ was observed for seven of nine patients with severe PLE amounts and for 15 of 19 patients with moderate to severe PLE (sensitivity $78 \%$ and specificity $49 \%$ ). The corresponding positive and negative predictive values were $40 \%$ and $84 \%$ for the detection of moderate to severe PLE.

In total, the mean reduction of PLE during follow-up was $0.8 \mathrm{~cm}$ (SD $2.1 \mathrm{~cm}$; range from -9.7 to $+0.7 \mathrm{~cm})$. For obvious reasons, the reduction of PLE was greatest among those with moderate to severe amount $(-2.6 \mathrm{~cm}$, SD 3.5, range from -9.7 to $+0.7 \mathrm{~cm}$ ) compared with those with only mild or no PLE $(-0.07 \mathrm{~cm}$, SD 0.2 , range from -1.0 to $0 \mathrm{~cm}$ ). During follow-up, the patients improved their MLHFQ-score by an average of 17 points (SD 27, range from -79 to +42 ). A total of 17 patients $(61 \%)$ had a clinically significant improvement in MLHFQ-score of $>5$ points reduction and $25(86 \%)$ 
Table 1 Baseline characteristics by the amount of pleural effusion

\begin{tabular}{|c|c|c|c|}
\hline Variables & $\begin{array}{l}\text { No or mild pleural } \\
\text { effusion }(n=43) \\
\text { mean } \pm S D \text { (range) }\end{array}$ & $\begin{array}{l}\text { Moderate to severe pleural } \\
\text { effusion }(\mathrm{N}=19) \\
\text { mean } \pm S D \text { (range) }\end{array}$ & p between groups \\
\hline Age, years & $74 \pm 12(35-91)$ & $75 \pm 11(49-92)$ & 0.69 \\
\hline Systolic blood pressure (mm Hg) & $125 \pm 24(85-171)$ & $115 \pm 18(80-150)$ & 0.12 \\
\hline Diastolic blood pressure (mm Hg) & $73 \pm 14(50-107)$ & $68 \pm 13(50-99)$ & 0.23 \\
\hline Heart rate $(\mathrm{bpm})$ & $75 \pm 18(51-121)$ & $88 \pm 26(56-140)$ & 0.02 \\
\hline NT-proBNP (ng/L) & $3252 \pm 2907$ (90-9999) & $5130 \pm 3320(93-9999)$ & 0.04 \\
\hline e-GFR $(\mathrm{ml} / \mathrm{min})$ & $51 \pm 20(16-88)$ & $53 \pm 23(26-99)$ & 0.73 \\
\hline Bumetanide-equivalent (mg) & $2.2 \pm 2.5(0-13)$ & $2.1 \pm 1.5(0-6)$ & 0.80 \\
\hline Weight & $85 \pm 19(56-141)$ & $75 \pm 18(49-120)$ & 0.05 \\
\hline MLHFQ-score & $\begin{array}{c}42 \pm 21(0-92) \\
\text { n (\%) }\end{array}$ & $\begin{array}{c}55 \pm 24(11-91) \\
\text { n (\%) }\end{array}$ & 0.03 \\
\hline Women & $20(47)$ & $10(53)$ & 0.66 \\
\hline Sinus rhythm (yes) & $21(49)$ & $4(21)$ & 0.04 \\
\hline \multicolumn{4}{|l|}{ Medication } \\
\hline Beta-blockers (yes) & $35(81)$ & $14(74)$ & 0.49 \\
\hline ACEI or ARB (yes) & $26(61)$ & $11(58)$ & 0.85 \\
\hline Patient-reported NYHA-class & & & 0.23 \\
\hline NYHA-class 1 & $3(7)$ & $1(5)$ & \\
\hline NYHA-class 2 & $24(56)$ & $6(32)$ & \\
\hline NYHA-class 3 & $9(21)$ & $5(26)$ & \\
\hline NYHA-class 4 & $7(16)$ & $7(37)$ & \\
\hline
\end{tabular}

patients reported an improvement in NYHA-class. There was no difference in the proportion of patients who experienced clinically meaningful improvement in MLHFQ-score by dichotomised PLE groups $(p=0.2)$. The improvement in MLHFQ-score correlated with improvement of NYHA-class $(\mathrm{r}=0.44, \mathrm{p}=0.02)$. We observed a linear improvement in the MLHFQ-score (3.2 points, $95 \%$ CI 1.2 to 5.1 ) with each $\mathrm{cm}$ reduction in PLE. This improvement in MLHFQ was not explained

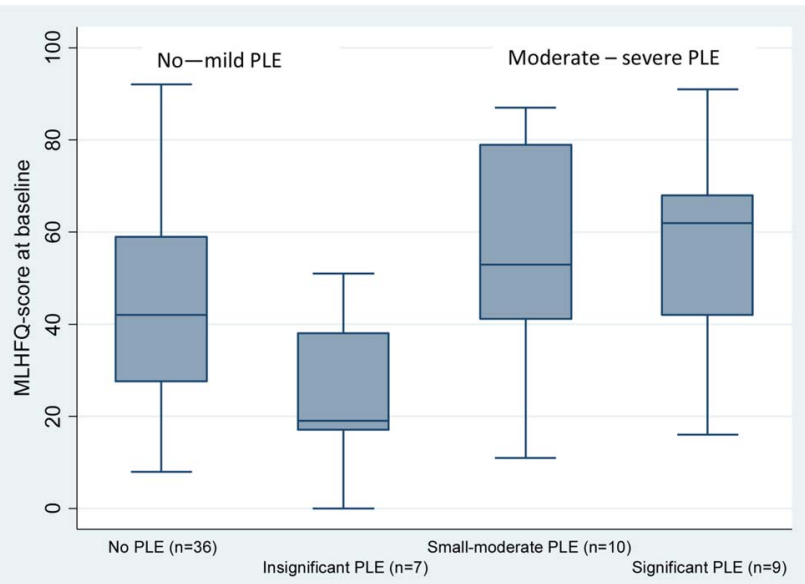

Figure 2 Shows the MLHFQ-score by amount of PLE in four categories at baseline. MLHFQ-score, Minnesota Living with Heart Failure Questionnaire score; PLE, pleural effusion. by any of the adjustments for age, sex, $\beta$-blockers, bumetanide-equivalent, ACEI/ARB, change in weight or change in blood pressure over time (see table 2). Figure 4 shows the linear two-way association of MLHFQ-score with the reduction of PLE over time. The odds for reduction in NYHA-class per $\mathrm{cm}$ reduction in PLE was 1.06 (95\% CI 1.04 to $1.08, \mathrm{p}<0.001)$ and none of the seven adjustments models explained the association (data not shown).

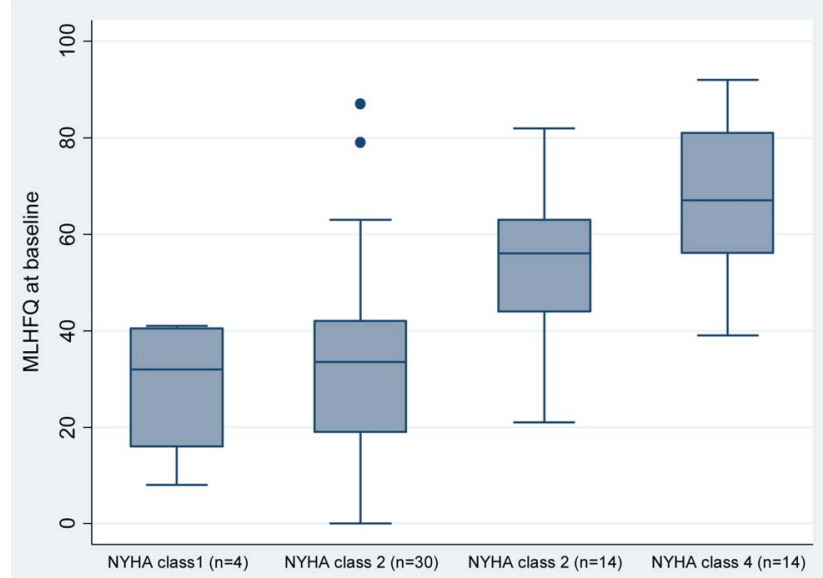

Figure 3 Shows MLHFQ-score by self-reported NYHA-class at baseline. MLHFQ-score, Minnesota Living with Heart Failure Questionnaire score; NYHA-class, New York Heart Association functional classification. 
Table 2 Change in patient-reported Minnesota Living with Heart Failure Questionnaire (MLHFQ)-score by decrease in pleural effusion $(\mathrm{cm})$

\begin{tabular}{|c|c|c|}
\hline Model & Adjustments & $\begin{array}{l}\text { B-coefficient } \\
(95 \% \mathrm{Cl})\end{array}$ \\
\hline Model 1 & Age & 3.1 (1.1 to 5.0$)$ \\
\hline Model 2 & Age + sex & 3.1 (1.0 to 5.2$)$ \\
\hline Model 3 & Model 1+ beta-blockers & 3.2 (1.3 to 5.2$)$ \\
\hline Model 4 & $\begin{array}{l}\text { Model } 1+\text { bumetanide } \\
\text { equivalent (in } \mathrm{mg} \text { ) }\end{array}$ & 3.1 (1.1 to 5.0$)$ \\
\hline Model 5 & $\begin{array}{l}\text { Model } 1+\text { weight } \\
\text { (change over time) }\end{array}$ & $3.2(0.8$ to 5.5$)$ \\
\hline Model 6 & Model $1+\mathrm{ACEl}$ or $\mathrm{ARB}$ & 3.1 (0.9 to 5.2$)$ \\
\hline Model 7 & $\begin{array}{l}\text { Model } 1+\text { systolic BP } \\
\text { (change over time) }\end{array}$ & $3.0(0.6$ to 5.5$)$ \\
\hline \multicolumn{3}{|c|}{$\begin{array}{l}\text { B indicates the decrease in MLHFQ-score per } \mathrm{cm} \text { decrease of } \\
\mathrm{PLE}(95 \% \mathrm{Cl}) \text { over time. } \\
\text { ACEI, angiotensin-converting enzyme inhibitor; } \mathrm{ARB} \text {, angiotensin } \\
\text { receptor blocker; } \mathrm{BP} \text {, blood pressure }(\mathrm{mm} \mathrm{Hg}) \text {. }\end{array}$} \\
\hline
\end{tabular}

\section{DISCUSSION}

Our study of outpatients with HF demonstrates that patient-reported QoL, by MLHFQ, is a sensitive tool for identifying patients with HF with severe amount of PLE. Furthermore, we found an improvement in MLHFQ-score and NYHA-class associated with reduction of PLE. A reduction of $1 \mathrm{~cm}$ of PLE corresponded to a reduction of 3.2 points in MLHFQ-score and 1.06 higher odds of improvement in functional class. In the models used, it seems that the change in PLE over time explains the improvement in MLHFQ-score and NYHA-class. Thus, paying attention to and treating PLE in follow-up of patients with HF may be important also with respect to improved symptoms and QoL.

To the best of our knowledge, no study has assessed the sensitivity of MLHFQ to detect PLE among

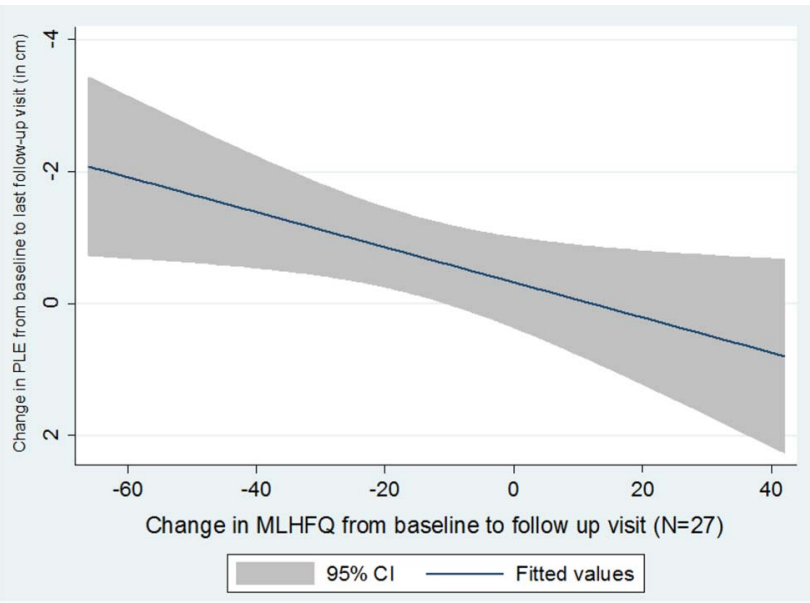

Figure 4 Shows change in MLHFQ-score by change in PLE graphed by linear regression approach. MLHFQ-score, Minnesota Living with Heart Failure Questionnaire score; PLE, pleural effusion. outpatients with HF. We find that the MLHFQ is a sensitive tool for detecting severe PLE in HF follow-up, and that a MLHFQ-score $>40$ should warrant further examination to search for PLE. The MLHFQ-score cut-off at 40 ensured that the impact on QoL for most of the patients with moderate-severe PLE was observed. However, half of the patients without PLE had MLHFQ-score $>40$ and treatment of PLE based solely on QoL is not advisable. However, as early detection of PLE is crucial for optimal follow-up of patients with HF, a moderate or worsened QoL should drive further diagnostic approach, and thereafter treatment. The MLHFQ-score is an easy and cheap method to raise the suspicion of PLE in patients with HF, and thus, should be routinely applied.

Our study is also the first to describe the associations of reduced amount of PLE with improved self-reported MLHFQ-score and NYHA-class among patients with HF. The relation between HF symptoms and patientreported outcomes is an area outlined in need of future research. ${ }^{19}$ The improvement in MLHFQ-score and NYHA-class were intercorrelated and in line with a previous study that showed improvement of MLHFQ-score during follow-up in a multidisciplinary HF programme. ${ }^{20}$

Point-of-care ultrasound of the pleural cavities is a sensitive and specific test for detection of PLE, ${ }^{121}$ and is previously shown to be superior to chest X-ray for diagnostics of PLE. ${ }^{22}$ The current study indicates that caring for improvement of patient-reported outcomes is an additional reason for systematic use of ultrasound to assess PLE in patients with $\mathrm{HF}^{21}$ as excessive volume status could be an explanation for the poor QoL. However, if the availability of ultrasound is limited, a MLHFQ-score $>40$ identifies $\sim 80 \%$ of those with moderate to severe PLE and these should be referred to ultrasound.

A determinant for the improvement in MLHFQ-score in an earlier study was found to be correlated with increased systolic blood pressure. ${ }^{20}$ In our study, adjustment for change in systolic blood pressure did not alter the improvement in MLHFQ-score associated with PLE reduction. However, the blood pressure was considered before titrating $\mathrm{HF}$ medication in order to prevent hypotension.

Respiratory symptoms have been reported to reduce QoL in patients with cancer ${ }^{23}$ and PLE is associated with respiratory symptoms in patients with $\mathrm{HF}^{2}{ }^{4}$ The observed improvement in QoL and NYHA-class may be influenced by the expected improvement in respiratory symptoms through other mechanisms causing dyspnoea in HF and not only by the PLE reduction per se. However, in palliative care, intermittent pleural drainage is often associated with superior palliation, improvement of $\mathrm{QoL}$ and decreased morbidity compared with no treatment of the PLE, ${ }^{92}$ and we observed a reduction of PLE to be copresent with improvement in symptoms following targeted treatment. 
Nevertheless, the study also has limitations. The study was conducted at a single HF outpatient clinic and the sample size was small with limited number of patients with severe HF and large PLE. However, the presented population is previously shown to be comparable with studies from other HF clinics with regard to HF severity, age, sex, NYHA-class and comorbidity and thus, it is plausible that our results are generalisable to other $\mathrm{HF}$ clinics. ${ }^{11}$ Assessment of HF symptoms is difficult because of the subjective nature of the symptoms. Incorporation of patient-reported outcome measures in clinical practice may be important to improve management and care for patients with HF. ${ }^{1325} 26$

Poor patient-reported MLHFQ-score and NYHA-classification are previously shown to be a strong predictor for early death in patients with HF. 202527 Improvement of MLHFQ-score during follow-up of HF is previously shown to predict event-free survival. ${ }^{20} 2829$ The low number of participants do not allow for survival analyses. However, self-report to detect PLE is useful as patients may prefer a better QoL over prolonged life. ${ }^{30}$

As patient-reported QoL is increasingly emphasised in treatment strategies, further studies should be performed in order to evaluate the need for more systematic identification and earlier intervention aiming to reduce PLE in follow-up of patients with HF.

\section{CONCLUSIONS}

The MLHFQ-score seems sensitive to detect severe PLE. Patient-reported outcome measures like the MLHFQ-score and NYHA-class were associated with the amount of PLE. Further, the long-term improvement in MLHFQ-score and NYHA-class were associated with reduced amount of PLE. Thus, reducing PLE may be important to improve the QoL in patients with HF. Routinely including patient-reported outcomes in follow-up of outpatients with HF may identify those at highest risk of having symptomatic amount of PLE and may allow for further improvement in the care for these patients. Further studies are needed to draw definitive conclusions regarding the interrelationship between PLE, QoL assessment and outcomes.

\footnotetext{
Author affiliations

${ }^{1}$ Department of Medicine, Levanger Hospital, Nord-Trøndelag Hospital Trust, Levanger, Norway

${ }^{2}$ Department of Heart Disease, Haukeland University Hospital, Bergen, Norway ${ }^{3}$ Department of Clinical Science, Faculty of Medicine and Dentistry, University of Bergen, Bergen, Norway

${ }^{4}$ Department of Neuromedicine (INM), Norwegian University of Science and Technology (NTNU), Trondheim, Norway

${ }^{5}$ Department of Circulation and Medical Imaging, Faculty of Medicine, Norwegian University of Science and Technology (NTNU), Trondheim, Norway ${ }^{6}$ Department of Circulation and Medical Imaging, Faculty of Medicine, K.G. Jebsen Centre of Exercise in Medicine, Norwegian University of Science and Technology (NTNU), Trondheim, Norway

${ }^{7}$ Department of Cardiology, St. Olavs University Hospital, Trondheim, Norway
}

Acknowledgements The Department of Internal Medicine, Levanger Hospital, at Nord-Trøndelag Hospital Trust, gave invaluable support in order to conduct and write up the study.

Contributors HD designed the study. All authors contributed to acquisition, analyses or interpretation of data. GHG and LTG drafted the manuscript, and all authors critically reviewed and approved submission of the final version of the manuscript.

Funding This study was funded by The Department for Research and Development at Nord-Trøndelag Hospital Trust.

Competing interests HD held a position at the Medical Imaging Laboratory, Norwegian University of Science and Technology (NTNU), a centre of research-based innovation that is funded by the Research Council of Norway and industry. One of the industry partners was GE Vingmed Ultrasound, which contributed to the total budget with 7 million NOK $(6 \%)$ for the 8-year period 2007-2014.

Ethics approval The Committee for Medical and Health Research Ethics (REK 2013/257)

Provenance and peer review Not commissioned; externally peer reviewed.

Data sharing statement No additional data are available.

Open Access This is an Open Access article distributed in accordance with the Creative Commons Attribution Non Commercial (CC BY-NC 4.0) license, which permits others to distribute, remix, adapt, build upon this work noncommercially, and license their derivative works on different terms, provided the original work is properly cited and the use is non-commercial. See: http:// creativecommons.org/licenses/by-nc/4.0/

\section{REFERENCES}

1. Porcel JM, Azzopardi M, Koegelenberg CF, et al. The diagnosis of pleural effusions. Expert Rev Respir Med 2015;9:801-15.

2. Freeman RK. Treatment options for patients with recurrent, symptomatic pleural effusions secondary to heart failure. Curr Opin Pulm Med 2015;21:363-7.

3. Kataoka H. Ultrasound pleural effusion sign as a useful marker for identifying heart failure worsening in established heart failure patients during follow-up. Congest Heart Fail 2012;18:272-7.

4. Porcel JM, Light RW. Pleural effusions. Dis Mon 2013;59:29-57.

5. Kato N, Kinugawa K, Seki S, et al. Quality of life as an independent predictor for cardiac events and death in patients with heart failure. Circ J 2011;75:1661-9.

6. Nieminen MS, Dickstein K, Fonseca C, et al. The patient perspective: quality of life in advanced heart failure with frequent hospitalisations. Int J Cardiol 2015;191:256-64.

7. Dunderdale K, Thompson DR, Miles JN, et al. Quality-of-life measurement in chronic heart failure: do we take account of the patient perspective? Eur J Heart Fail 2005;7:572-82.

8. Lenker A, Mayer DK, Bernard SA. Interventions to treat malignant pleural effusions. Clin J Oncol Nurs 2015;19:501-4.

9. Lorenzo MJ, Modesto M, Perez J, et al. Quality-of-life assessment in malignant pleural effusion treated with indwelling pleural catheter: a prospective study. Palliat Med 2014;28:326-34.

10. Dalen H, Gundersen GH, Skjetne K, et al. Feasibility and reliability of pocket-size ultrasound examinations of the pleural cavities and vena cava inferior performed by nurses in an outpatient heart failure clinic. Eur J Cardiovasc Nurs 2015;14:286-93.

11. Gundersen GH, Norekval TM, Haug HH, et al. Adding point-of-care ultrasound to assess volume status in heart failure patients in a nurse-led outpatient clinic. A randomised study. Heart 2016;102:29-34.

12. McMurray JJ, Adamopoulos S, Anker SD, et al. ESC Guidelines for the diagnosis and treatment of acute and chronic heart failure 2012: the Task Force for the Diagnosis and Treatment of Acute and Chronic Heart Failure 2012 of the European Society of Cardiology. Eur Heart J 2012;33:1787-847.

13. Anker SD, Agewall S, Borggrefe M, et al. The importance of patient-reported outcomes: a call for their comprehensive integration in cardiovascular clinical trials. Eur Heart J 2014;35:2001-9.

14. Holland R, Rechel B, Stepien K, et al. Patients' self-assessed functional status in heart failure by New York Heart Association class: a prognostic predictor of hospitalizations, quality of life and death. J Card Fail 2010;16:150-6. 
15. Morken IM, Isaksen K, Karlsen B, et al. Shock anxiety among implantable cardioverter defibrillator recipients with recent tachyarrhythmia. Pacing Clin Electrophysiology 2012;35: 1369-76.

16. Rector TS, Kubo SH, Cohn JN. Patients' self-assessment of their congestive heart failure. Content, reliability and validity of a new measure, The Minnesota Living with Heart Failure Questionnaire. Heart Fail 1987:198-209.

17. Behlouli $\mathrm{H}$, Feldman DE, Ducharme A, et al. Identifying relative cut-off scores with neural networks for interpretation of the Minnesota Living with Heart Failure questionnaire. Conf Proc IEEE Eng Med Biol Soc 2009;2009:6242-6.

18. Matsushita K, Mahmoodi BK, Woodward M, et al. Comparison of risk prediction using the CKD-EPI equation and the MDRD study equation for estimated glomerular filtration rate. JAMA 2012;307:1941-51.

19. Zannad F, Garcia AA, Anker SD, et al. Clinical outcome endpoints in heart failure trials: a European Society of Cardiology Heart Failure Association consensus document. Eur J Heart Fail 2013;15: 1082-94.

20. Hole T, Grundtvig M, Gullestad L, et al. Improved quality of life in Norwegian heart failure patients after follow-up in outpatient heart failure clinics: results from the Norwegian Heart Failure Registry. Eur J Heart Fail 2010;12:1247-52.

21. Ponikowski P, Voors AA, Anker SD, et al. 2016 ESC Guidelines for the diagnosis and treatment of acute and chronic heart failure: the task force for the diagnosis and treatment of acute and chronic heart failure of the European Society of Cardiology (ESC). Eur J Heart Fail 2016;18:891-975.
22. Graven T, Wahba A, Hammer AM, et al. Focused ultrasound of the pleural cavities and the pericardium by nurses after cardiac surgery. Scand Cardiovasc J 2015;49:56-63.

23. Neumann K, Sundset A, Espinoza A, et al. Changes in quality of life, dyspnea scores, and lung function in lung cancer patients with airway obstruction after a therapeutic bronchoscopy. J Bronchology Interv Pulmonol 2013;20:134-9.

24. Sabur NF, Chee A, Stather DR, et al. The impact of tunneled pleural catheters on the quality of life of patients with malignant pleural effusions. Respiration 2013;85:36-42.

25. Mastenbroek $\mathrm{MH}$, Versteeg $\mathrm{H}$, Zijlstra $\mathrm{W}$, et al. Disease-specific health status as a predictor of mortality in patients with heart failure: a systematic literature review and meta-analysis of prospective cohort studies. Eur J Heart Fail 2014;16:384-93.

26. Norekval TM, Falun N, Fridlund B. Patient-reported outcomes on the agenda in cardiovascular clinical practice. Eur $J$ Cardiovasc Nurs 2016;15:108-11.

27. Hoekstra T, Jaarsma T, van Veldhuisen DJ, et al. Quality of life and survival in patients with heart failure. Eur J Heart Fail 2013;15:94-102.

28. Moser DK, Yamokoski L, Sun JL, et al. Improvement in health-related quality of life after hospitalization predicts event-free survival in patients with advanced heart failure. J Card Fail 2009;15:763-9.

29. Soriano N, Ribera A, Marsal JR, et al. Improvements in health-related quality of life of patients admitted for heart failure. The HF-QoL study. Rev Esp Cardiol 2010;63:668-76.

30. Lewis EF, Johnson PA, Johnson W, et al. Preferences for quality of life or survival expressed by patients with heart failure. J Heart Lung Transplant 2001;20:1016-24. 